\title{
Ciencia y evolución del dióxido de zirconio, de la prioridad mecánica a la necesidad estética
}

\author{
Science and evolution of zirconium dioxide, from mechanical priority to aesthetic need \\ Jesús Marcelo ${ }^{1, a}$, Gerardo Miguel Gallet-Alfaro $^{1,2, b, c}$, Leila Maritza Fernández-Jacinto ${ }^{1,2, a, b}$, Diego Hinostroza-Noreña ${ }^{1, a}$
}

\begin{abstract}
RESUMEN
En la actualidad, los procedimientos de alta tecnología enfocados a las restauraciones en base a cerámicas dentales han permitido la evolución y perfeccionamiento de distintas formas de manufactura de restauraciones, iniciándose así, la utilización de cerámicas como el dióxido de zirconio. Objetivo: Identificar las publicaciones recientes de los materiales de restauración a base de dióxido de zirconio que incluyan estudios clínicos, propiedades mecánicas, físicas y estéticas, así como su evolución, manufactura y diversas aplicaciones en la práctica clínica diaria. Material y métodos: La búsqueda sistemática de la literatura se realizará en las bases de datos de PubMed y Ebsco, se considerarán publicaciones del 20102019 y que fueran investigaciones clínicas, laboratoriales y todas aquellas que mostraban la evolución en el tiempo del dióxido de zirconio, así como su aplicabilidad y diversificación. Resultados: Se revisaron 48 artículos que cumplieron con los criterios de selección para la revisión bibliográfica. Se encontró que todas las restauraciones protésicas a base de dióxido de zirconio, indistintamente a su forma de utilización, mostraron un desempeño clínico muy similar a los tres años, después de ese tiempo, dependerá del tipo de utilización, composición y elección del caso. Conclusiones: El éxito clínico de las restauraciones en base a dióxido de zirconio, es debido a las múltiples ventajas mecánicas y, con las recientes modificaciones estructurales, sumadas al agregado de aditivos para su estabilización, se perfila como un material idóneo para el trabajo tanto en zonas estéticas como en áreas de necesidad de soporte mecánico con carga funcional elevada. PALABRAS CLAVE: Coronas, circonio, porcelana dental, diseño asistido por computador.
\end{abstract}

\section{SUMMARY}

At present, high-tech procedures focused on restorations based on dental ceramics have allowed the evolution and improvement of different forms of manufacturing restorations, thus initiating the use of ceramics such as zirconium dioxide. Objective: To identify recent publications of zirconium dioxide based restoration materials that include clinical studies, mechanical, physical and aesthetic properties, as well as their evolution, manufacturing and various applications in daily clinical practice. Materials and methods: The systematic search of the literature will be carried out in the databases of PubMed and Ebsco, publications from 2010-2019 will be considered and that they were clinical, laboratory research and all those that showed the evolution in time of zirconium dioxide, as well as its applicability and diversification. Results: 48 articles were reviewed that met the selection criteria for the literature review. It was found that all prosthetic restorations based on zirconium dioxide, regardless of their use, showed a clinical performance very similar to three years, after that time, will depend on the type of use, composition and choice of case. Conclusions: The clinical success of restorations based on zirconium dioxide is due to the multiple mechanical advantages and, with the recent structural modifications, added to the addition of additives for stabilization, is emerging as an ideal material for work both aesthetic areas as in areas of need for mechanical support with high functional load.

KEYWORDS: Crowns, zirconium, dental porcelain, computer-aided design.

Facultad de Estomatología Roberto Beltrán, Universidad Peruana Cayetano Heredia. Lima, Perú. Departamento de Clínica Estomatológica de la Universidad Peruana Cayetano Heredia. Lima, Perú.

Diplomado en Rehabilitación Oral.

Docente Auxiliar

Especialista en Rehabilitación Oral. 


\section{INTRODUCCIÓN}

Con la necesidad de implementar mejoras, tanto mecánicas como estéticas, las restauraciones de cobertura completa están en constante evolución; esta evolución a los que fueron sometidos y a la introducción de nuevos sistemas cerámicos libres de metal en la odontología moderna han provocado que las ventajas e indicaciones de estos materiales cerámicos se expandan a mayor número de situaciones (1), algunas de ellas impensadas hace pocos años por lo que han ganado una gran popularidad en odontología. Aunque estudios previos de las propiedades mecánicas y ópticas de los materiales cerámicos actuales indican que ningún sistema cerámico es ideal para todas las situaciones clínicas $(2,3)$. Pese a que las coronas metalocerámicas aún son catalogadas como el "gold estándar" por sus excelentes propiedades físicas (garantizando un adecuado funcionamiento mecánico) y alta predictibilidad (asegurando su supervivencia en el tiempo), presentan desventajas estéticas como el margen metálico que suele dificultar la mimetización con los dientes naturales, especialmente en los biotipos periodontales delgados donde los problemas de recesión y transparencia resultan en un halo gris azulado en los tejidos blandos periféricos $(3,4)$.

Otro inconveniente alrededor de estas restauraciones tienen que ver con el espacio necesario que requieren para sus componentes $(\geq 2 \mathrm{~mm})$, que posteriormente fue resuelto con el desarrollo de un diseño de la estructura reducida y nuevas cerámicas de hombro (5). Así pues, las coronas libres de metal surgieron con la finalidad de lograr mejores resultados estéticos, además de resolver otros problemas como el galvanismo y la toxicidad $(1,6)$. En la actualidad, muchos materiales cerámicos que aparecieron para contrarrestar las deficiencias de las prótesis metalcerámicas han ido aportando una serie de mejoras en lo que a estética y resistencia respecta $(5,6)$.

Las cerámicas dentales son calificadas como materia prima de naturaleza inorgánica, teniendo mayor porcentaje de elementos no metálicos que se obtienen a partir de la generación de calor (mayormente combustión) (7). En general, se caracterizan por ser biocompatibles, resistentes a la corrosión, no reaccionan con líquidos, ni ácidos; aunque en un principio no presentaban una buena resistencia a la flexión, tracción y fractura, las cerámicas actuales han ido superando dichas desventajas (5). La característica primordial que permite utilizarlas como material restaurador, es su naturaleza refractaria y sus propiedades ópticas, además de su excelente biocompatibilidad $(2,3,4,7)$. En lo que respecta a su composición cuenta con 2 fases: una fase amorfa o matriz, que otorga propiedades estéticas y una fase cristalina que otorga propiedades mecánicas (5). Las cerámicas cristalinas son generalmente más fuertes que la cerámica de vidrio, que permiten el paso de luz a través de él, lo que hace que sea translúcida. Las cerámicas de cristal, por el contrario, suelen ser opacas debido a las discrepancias de índices de refracción. En resumen, el mayor contenido de cristal en una cerámica contribuye en general a una mayor resistencia y disminución de la translucidez (3).

Con el pasar de los años, especialmente las últimas décadas, los procedimientos tecnológicos avanzados aplicados a las porcelanas dentales han guiado el desarrollo de distintas formas de manufactura de restauraciones, tales como porcelanas prensadas, inyectadas, infiltradas y maquinadas o torneadas $(5$, 6). Es así, que se inicia la utilización de cerámicas como el dióxido de zirconio; el zirconio, como elemento, está incluido en el grupo de los metales de la tabla periódica, por ello va a gozar de las características del metal en cuanto a la resistencia, comportamiento químico y óptico, esta cualidad es interesante cuando se quiere enmascarar un sustrato oscuro, diversos aditivos se han ido utilizando para mejorar las propiedades de este tipo de cerámica, como el dióxido de itrio para estabilizar su fase molecular tetragonal $(6,9)$. Siendo este metal, ya utilizado por más de 15 años por los laboratorios para restauraciones dentales indirectas como subestructura para otras cerámicas de recubrimiento o de manera totalmente anatómica (4).

Las restauraciones dentales fijas basadas en dióxido de zirconio estabilizado con óxido de itrio (Y-TZP) se han convertido en populares gracias a sus propiedades mecánicas favorables, excelente biocompatibilidad y buena estética cuando son recubiertas con cerámica feldespática (10). Un creciente número de estudios sobre las restauraciones basadas en dióxido de zirconio muestran tasas de supervivencia a corto plazo similares a las restauraciones de cerámica sobre metal $(95,9$ $98,5 \%$ después de 5 años, pero disminuye en 10 años a $67.2 \%$ ); siendo el factor más común de fracaso la 
fractura del material de recubrimiento o su astillamiento (8). Aunque no todas las fracturas han dado lugar a la eliminación de las restauraciones, se ha observado que las fracturas predominantemente cohesivas del material de recubrimiento, todavía presentan un problema que necesita ser evaluado $(5,7,10)$.

Una forma de evitar las fracturas del material de recubrimiento es excluirla y en su lugar utilizar coronas monolíticas obtenidas exclusivamente con el material del núcleo de alta resistencia. Esto es posible mediante el uso de CAD/CAM, donde las coronas monolíticas se pueden fresar en su totalidad con su contorno anatómico. En lugar de la construcción de la porcelana en varias capas y disparando en múltiples ciclos de cocción, la corona monolítica puede ser individualizada $(4,6,10)$.

El objetivo de esta revisión es identificar las características del dióxido de zirconio monolítico, así como su aplicabilidad a la odontología restauradora actual, clasificándolas según su composición y método de fabricación. De la misma forma, se describirá desde la perspectiva mecánica, estética y funcional la presencia de esta cerámica en la práctica diaria profesional. Por lo antes mencionado, el objetivo del presente artículo de revisión fue identificar las publicaciones recientes de los materiales de restauración a base de óxido de zirconio que cumplan e incluyan estudios clínicos, propiedades mecánicas, físicas y estéticas, así como su evolución, manufactura y diversas aplicaciones en la práctica clínica diaria. La búsqueda sistemática se realizó en las bases de datos de PubMed y Ebsco.

\section{MATERIAL Y METODOS}

El presente trabajo tuvo como objetivo una revisión sistemática de la literatura científica. La revisión se realizó en las bases de datos de PubMed (MedLine) y Ebsco. La búsqueda se efectuó con las siguientes palabras clave o key words: Coronas, Circonio, Porcelana dental, Diseño Asistido por Computador, Crowns, Zirconium, Dental porcelain, ComputerAided Design, respectivamente. Los criterios de selección de los artículos fueron artículos publicados entre el 2010-2019 y que fueran investigaciones clínicas, laboratoriales y todas aquellas que mostraban la evolución en el tiempo del dióxido de zirconio, así como su aplicabilidad y diversificación. El desarrollo de la revisión consistió en antecedentes, indicaciones, contraindicaciones, ventajas, desventajas, propiedades mecánicas, físicas y estéticas; además fue centrado en la revisión de los estudios clínicos e in vitro de las restauraciones dentales a base de dióxido de zirconio.

\section{Dióxido de zirconio ( $\mathrm{ZrO2})$}

Fue el químico Klaproth el que aisló por primera vez el dióxido de zirconio o zirconia $(\mathrm{ZrO} 2)$ en el año 1798; este material lo podemos encontrar presente en la naturaleza en su fase monoclínica (densidad más baja), existiendo otras dos fases cristalinas, que dependen de la temperatura para su transformación $(2,8)$. Al calentar la zirconia a $1170^{\circ} \mathrm{C}$, la fase monoclínica se transforma en fase tetragonal, la que proporciona mejores propiedades mecánicas, la fase tetragonal se transforma en la fase cúbica a $2370^{\circ} \mathrm{C}$, esta fase tiene propiedades mecánicas moderadas y es estable hasta $2680^{\circ} \mathrm{C}$ (figura 1), donde pasa a fundirse; el paso de la fase tetragonal
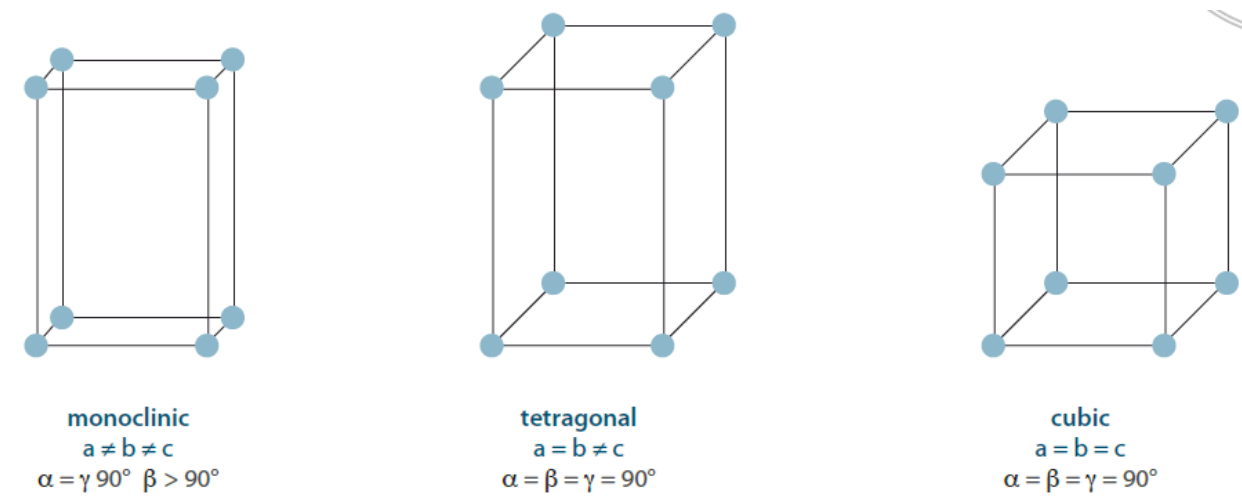

Figura 1. Dióxido de zirconio en sus tres fases (8). 
a la monoclínica, que es la más discutida por la alteración de sus propiedades, también se describe como "transformación martensítica" y se caracteriza por un aumento en el volumen de aproximadamente el $4 \%$, que puede conllevar a una alteración entre las fuerzas de tracción moleculares y hacerlas menos densas ocasionando grietas estructurales dentro del material $(3,7)$.

La fase más ventajosa para el trabajo odontológico es la tetragonal, pero ésta al no ser estable a temperatura ambiente después de los cambios físicos que sufre la materia (calentamiento y/o enfriamiento), necesita de óxidos estabilizadores para los cristales de zirconia $\left(\mathrm{CaO}, \mathrm{MgO}, \mathrm{CeO} 2, \mathrm{Y}_{2} \mathrm{O}_{3}\right)$; sin embargo, debido a que la fase tetragonal solo se estabiliza parcialmente, la transformación desfavorable de dicha fase a la monoclínica puede ocurrir cuando se proporciona algún tipo de energía externa, y producir algún cambio molecular entre las redes cristalinas $(3,7)$.

La fase cristalina o de relleno de la zirconia aporta resistencia mecánica con sus átomos

geométricamente ordenados, puesto que hacen de barrera a las microgrietas que se originan durante el procesado, o con el tiempo tras las cargas oclusales y la presencia de humedad $(5,7)$.

Para la transformación del dióxido de zirconio a sus fases cúbica y tetragonal es necesario la disminución de la temperatura después de la fusión del material, con la finalidad de que la estructura dimensional sufra cambios a nivel molecular, siendo estos cambios físicos críticos, debido a la posibilidad de producir grietas dentro de la red cristalina, éstas generan grandes tensiones que, normalmente pueden llevar al dióxido de zirconio puro a la fractura (11); entonces, la microestructura de la cerámica va a determinar sus propiedades mecánicas y ópticas. Las cerámicas cristalinas tienen átomos dispuestos en cristales muy juntos con una alta densidad atómica; mientras que los vidrios tienen una densidad atómica menor, con variantes en concentraciones de ambas para combinar sus propiedades (figura 2) (6), por lo tanto, una grieta que se propaga a través de una cerámica cristalina tendrá que romper más enlaces atómicos por unidad de área que una grieta que viaja a través de la misma unidad de superficie de una cerámica de vidrio, además de la densidad atómica inferior del vidrio (9). Se ha descubierto que este fenómeno se puede contrarrestar, añadiendo alguno de los siguientes óxidos al compuesto: Magnesia $(\mathrm{MgO})$, Itria $\left(\mathrm{Y}_{2} \mathrm{O}_{3}\right)$, Ceria $\left(\mathrm{Ce}_{2} \mathrm{O}_{3}\right) \mathrm{u}$ otros aditivos (10). El agregado de 2 a 3\% de óxido de itrio estabiliza parcialmente la fase tetragonal. Las coronas de zirconia parcialmente estabilizada con itria muestran propiedades mecánicas superiores, debido a valores de resistencia a la flexión y la resistencia a la fractura de $1600 \mathrm{MPa}$ y $9 \mathrm{MPa} /$ $\mathrm{m} 1 / 2$ respectivamente $(2,3,6)$.

A principios de 1990, el dióxido de zirconio tetragonal policristalino parcialmente estabilizado con óxido de itrio (Y-TZP) fue introducido en la odontología como material básico para las restauraciones de cerámica y ha sido puesto a disposición a través de la tecnología CAD/CAM. En comparación con otros sistemas de cerámica sin metal, el dióxido de zirconio exhibe propiedades mecánicas superiores, debido a

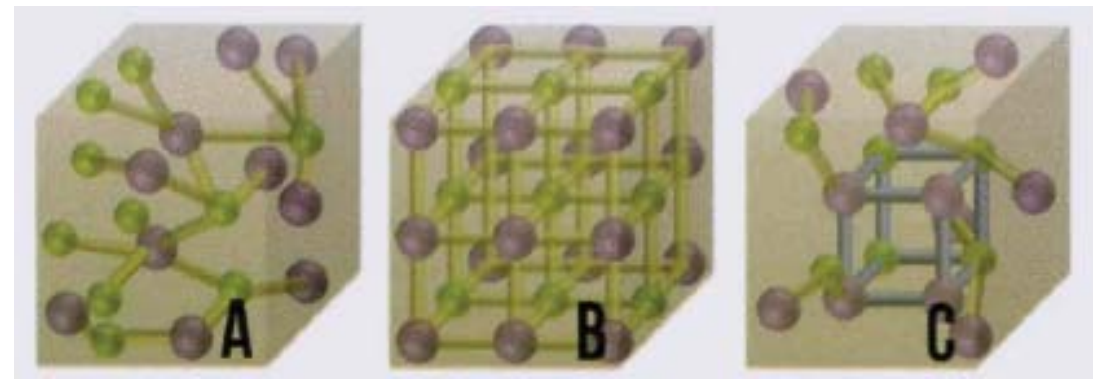

Figura 2. Microestructura de vidrio, (A) microestructura de cristal (B) y microestructura de cerámica parcialmente cristalina $(\mathrm{C})$. Esferas representan elementos metálicos y no metálicos, cadenas representan enlaces químicos (5). 
un mecanismo de resistencia a la deformación (8). La zirconia convencional o de primera generación (3Y-TZP) tiene un alto índice de refracción de la luz debido a las numerosas estructuras cristalinas muy pequeñas a través de las cuales tiene que pasar la luz, lo que genera el carácter opaco para el material, teniendo una composición básicamente de $\mathrm{Zr}_{2} \mathrm{O}$ $(>98 \%), \mathrm{Y}_{2} \mathrm{O}_{3}(\approx 4.5 \%)$ y $\mathrm{Al}_{2} \mathrm{O}^{3}(\approx 0.25 \%)(1)$.

De los materiales cerámicos sin metal disponibles en la actualidad, la zirconia parcialmente estabilizada con itria (3Y-TZP) presenta la mayor resistencia a la fractura y son comparables al gold standar en las restauraciones de coronas dentales $(4,7)$. Las coronas de 3Y-TZP se han utilizado tanto para restauraciones anteriores y posteriores, aunque la resistencia del

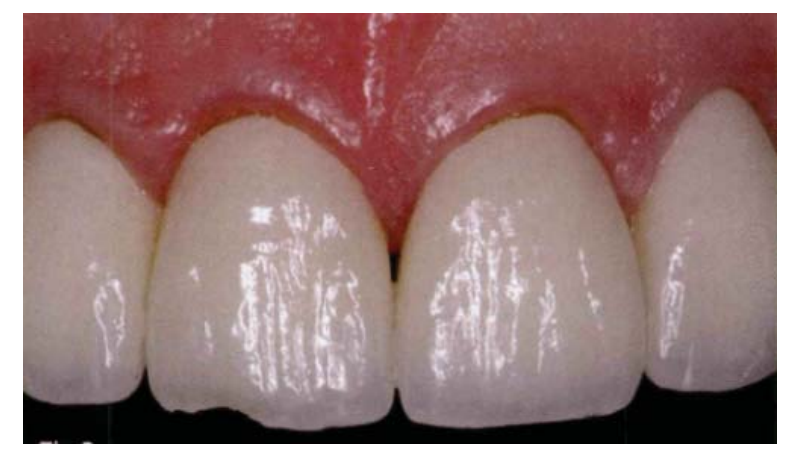

Figura 3. Fractura de cerámica de recubrimiento con núcleo de Y-TZP (anterior) (5). núcleo en estas coronas es excelente y las tasas de supervivencia son altos, algunos ensayos clínicos informan fracturas del material de recubrimiento (figura 3, figura 4 y figura 5), lo que reduce las tasas de éxito entre un $8 \%$ - $25 \%$ en un tiempo de $24-38$ meses $(6,8,10)$. Un punto importante a tener en cuenta sobre estas cerámicas de recubrimiento es, conocer su coeficiente de expansión térmica (CTE) ya que, estas coberturas deben tener un coeficiente menor que el núcleo para evitar su deformación debido a las veces que ingresa al horno para su estratificación o inyección; es aquí donde los cristales (zirconia), molecularmente tienen una participación importante ya que tienen un CTE mayor que los vidrios (feldespato) (6), otro factor desfavorable para ambas cerámicas es que no tienen ductilidad $\mathrm{y}$, por

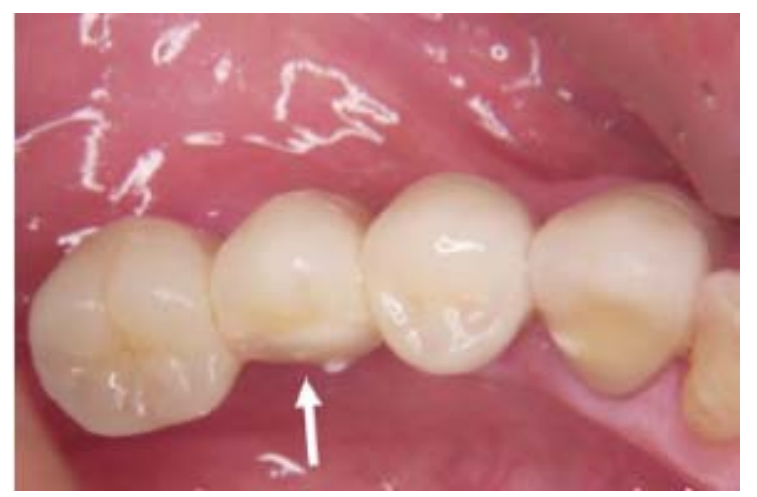

Figura 4. Fractura de cerámica de recubrimiento con núcleo de Y-TZP (posterior)(6).

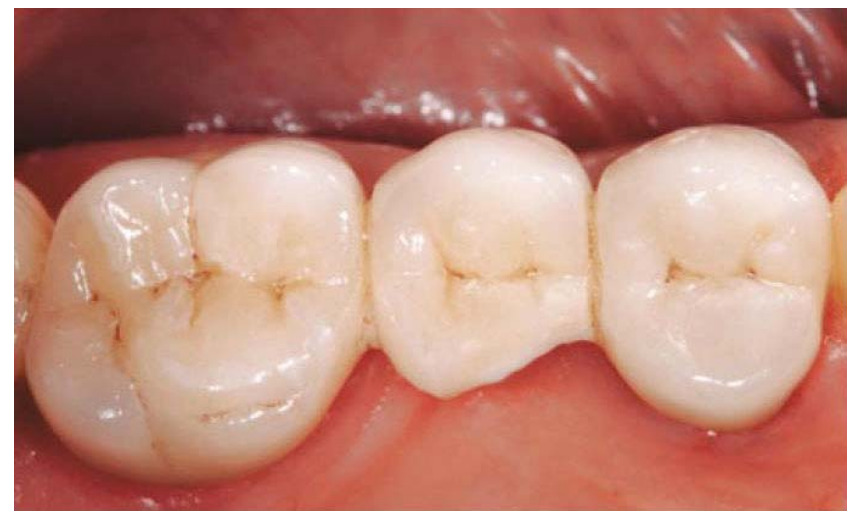

Figura 5. Fractura de cerámica de recubrimiento de puente fijo de 3 piezas después de 12 meses de cementado (8). 
lo tanto, no pueden compensar las tensiones internas (diferentes CTEs entre el núcleo y el recubrimiento) y externas (fuerzas de masticación), siendo la primera afectada la que menos resistencias flexurales presenta, traduciéndose en fracturas estructurales (11).

Actualmente este tipo de zirconia (primera generación), con alto índice refractario y numerosas interfaces cristalinas estructurales que crean el carácter opaco del material, se encuentra disponible en el mercado como: zirconia parcialmente estabilizada con magnesia, zirconia reforzada con alúmina y zirconia parcialmente estabilizada con itria (10).

\section{Dióxido de zirconio monolítico}

Surge la necesidad de utilizar un material más compacto con la suficiente resistencia para evitar los fallos por fractura de las cerámicas de cobertura, en consecuencia se inicia experimentando con los bloques de cerámica monolíticas $(3,7,11)$. Aquí, es donde se inicia la búsqueda de un material con mejores propiedades ópticas, descubriendo que, al disminuir el contenido de $\mathrm{Al}_{2} \mathrm{O}_{3}$, aumentaba la translucidez del dióxido de zirconio (de $\approx 0.25 \% \%$ a $\approx 0.05 \%$ ), denominándola zirconia de segunda generación (3Y-TZP); donde se mantenía la estructura cristalográfica tretragonal de la cerámica, y las propiedades mecánicas entre una y otra generación, no tenían diferencia estadísticamente significativa $(5,8)$.

La zirconia monolítica se ha utilizado ampliamente en la práctica clínica para restauraciones únicas y múltiples, como son: coronas y puentes sobre piezas dentarias, pilares de implantes, prótesis sobre implantes de arco completo, y hasta postes del canal radicular, debido a que es menos propenso a la fractura o astillamiento, que la cerámica feldespática vítrea de recubrimiento. 8 Propiedades como la resistencia a la fractura, flexión y tracción mejoran con la utilización de cerámicas monolíticas, evitando limitación en los grosores para su posterior estratificación; dichas restauraciones están casi directamente expuestas al ambiente oral, y requieren poco o ningún recubrimiento estético. Por lo tanto, la ausencia de estratificado superficial convencional (múltiples capas) elimina el problema de astillado o fractura $(2,3)$, proporcionando una mejor estabilidad a largo plazo y menor sensibilidad para el envejecimiento y los efectos de la fatiga, lo que puede ser ventajoso en condiciones clínicas (7), lo que no significa que sean totalmente infalibles o seguras, ya que también se han reportado fracturas de éstas coronas, pero con la limitante del grosor del material (figura 6) (3).

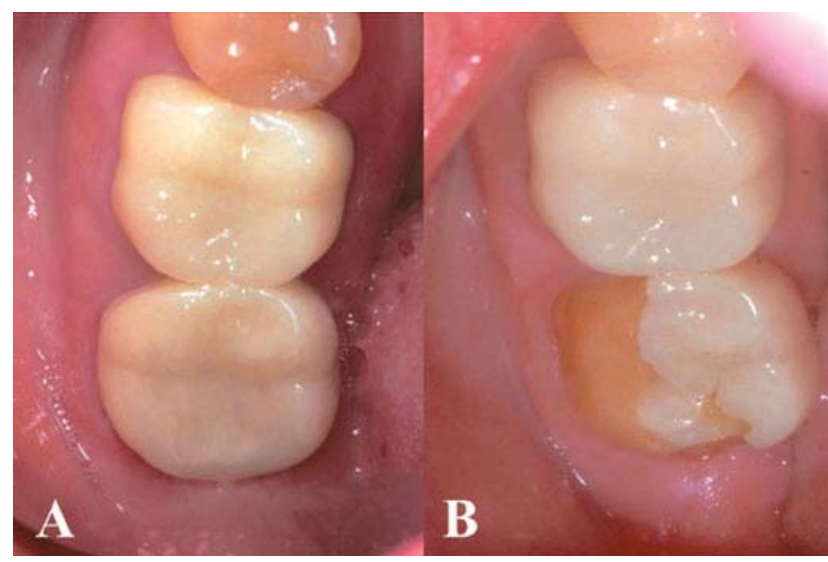

Figura 6. Restauración de zirconia monolítica fracturada. (A) Restauración cementada y (B) Control a los 38 meses de cementada (2).

Con las ventajas mecánicas logradas, empezó la preocupación por la estética, ya que la zirconia seguía siendo opaca y totalmente refractaria; es así, que el proceso de translucidez empezó a tener relevancia. Para tal efecto se empezó con la zirconia de primera generación, descubriendo que al aumentar la temperatura $\left(>1600^{\circ} \mathrm{c}\right)$ y el tiempo de sinterización se lograba cierta translucidez del material, pero resultantes negativas con respecto a la resistencia y a la estabilidad a largo plazo $(2,5)$. Es así, que se llevó a cabo una modificación a nivel molecular (3Y-TZP) conocida como zirconia de segunda generación, donde el número y el tamaño de los granos de óxido de aluminio (A12O3) se redujeron y reubicaron en la matriz de zirconia, esto significó una mayor transmisión de luz con una estabilidad a largo plazo consistentemente buena y alta resistencia $(6,8)$.

En el sector anterior, las coronas monolíticas hechas de materiales vitrocerámicos basados en disilicato de litio han demostrado una resistencia comparable a las metalocerámicas, teniendo en cuenta que los vectores de fuerza a las que están sometidas estas piezas son diferentes a las posteriores, y con el plus de una apariencia más natural y translúcida.10 Para el sector posterior, donde se ejercen mayores fuerzas axiales compresivas, las coronas monolíticas de disilicato de litio son una opción válida, pero en brechas cortas con 
algunas limitaciones como la parafunción y el tipo de antagonista; entonces surge la opción de usar zirconia monolítica para mejorar las propiedades mecánicas de los materiales restauradores $(8,11)$, el método de fabricación hace que la precisión del sistema $\mathrm{CAD} / \mathrm{CAM}$ sea cada vez más influyente y decisivo para la elaboración de estas cerámicas monolíticas, y estarán en relación directa al éxito clínico de las restauraciones (figura 7$)(2,9)$.

\section{Ventajas $(2,4,8,11)$}

Reducción del tiempo de producción en laboratorio. Mejora la resistencia, evitando fracturas.

Desgaste mínimo de antagonista (abrasión), inferior a otros materiales cerámicos.

Mejora de la rentabilidad.

Preparación mínimamente invasiva $(0,5-0,7 \mathrm{~mm})$.

Reduce tasa de complicaciones biológicas.

Ausencia de cerámica de recubrimiento.

Estética no comprometida (maquillaje o zirconia translúcida).

\section{Desventajas $(1,3,8)$}

Parcial: Cementación (adhesiva).

Costo en comparación a otras alternativas.

Espacio para conectores en caso de prótesis fija.

\section{Indicaciones $(4,7)$}

Poco espacio protésico en coronas.

Necesidad de alta resistencia.

Muñones cortos.

Alergia a componentes metálicos.

\section{Contraindicaciones $(6,9,11)$}

Imposibilidad de cumplir con protocolos de cementación.

Pacientes disfuncionados no controlados

Pacientes no estabilizados oclusal o periodontalmente.

En la actualidad no hay suficiente evidencia clínica a largo plazo del comportamiento de las restauraciones de zirconia monolítico enteramente anatómico, solo tenemos casos de seguimientos de hasta 4-5 años aproximadamente, sin embargo los riesgos potenciales de su aplicación clínica y las posibles ventajas están siendo evaluados íntegramente en estudios in vitro $(4,7,11)$.

\section{Dióxido de zirconio monolítico translúcido}

La nueva manera de enfrentar las fracturas de los materiales cerámicos utilizando las formas monolíticas aún tenían la limitante de la estética estricta, ya que no se contaba con muchas opciones en la gama de tonalidades $\mathrm{y}$, aunque se trataba de mimetizarlas mediante la técnica de maquillaje no era siempre suficiente; ante esta limitante, una nueva modificación, esta vez a nivel molecular de su fase cristalina estructural, 6 dio paso a un dióxido de zirconio translúcido, que al variar su composición química presentaba cierta disminución en la resistencia mecánica de la porcelana, aunque parece no tener desventajas clínicamente significativas, las recomendaciones de uso son limitadas a casos unitarios monolíticos $(4,9)$, este novedoso material fue sintetizado a partir del aumento en contenido y tamaño de los granos del Y2O3 $(\approx 4.5 \%$ segunda generación a $<10 \%$ tercera generación), teniendo como consecuencia un aumento de la translucidez y disminución de la resistencia $(9,13)$.

Esta zirconia de tercera generación (5Y-TZP) no solo es metaestable en la fase tetragonal (47\%), sino que también contiene una proporción de fase cúbica de hasta el $53 \%$ (8). Por lo tanto, se describe como "zirconia totalmente estabilizada con una estructura mixta cúbica/tetragonal". Las porciones cúbicas se lograron por un aumento de óxido de itrio (aprox. $8.8 \%-9.3 \%$ en peso / $5 \%$ en moles $)(1,7)$.

Los cristales cúbicos tienen mayor volumen que los tetragonales, ocasionando que la luz se disperse con menos fuerza en los límites del grano y las porosidades residuales, lo que hace que el material sea más translúcido (6); además, las estructuras de cristal cúbico son más isotrópicas, haciendo que la luz incidente se emita de manera más uniforme en todas las direcciones espaciales. Esta propiedad también tiene una influencia significativa en la translucidez (3, 7,11 ) (figura 8).

Entonces, el aumento en el contenido de óxido de itrio (5Y-TZP), donde se agrega una fase cúbica a la tetragonal metaestable mejora la translucidez de la zirconia (figura 9); sin embargo, la reducción de la fase tetragonal causa una disminución en la transformación molecular normal afectando negativamente las propiedades mecánicas (10), esto a consecuencia de lo que ocurre con las partículas de $\mathrm{Al}_{2} \mathrm{O}_{3}$ que normalmente aumentan dichas propiedades de la zirconia, pero al reducirse considerablemente 

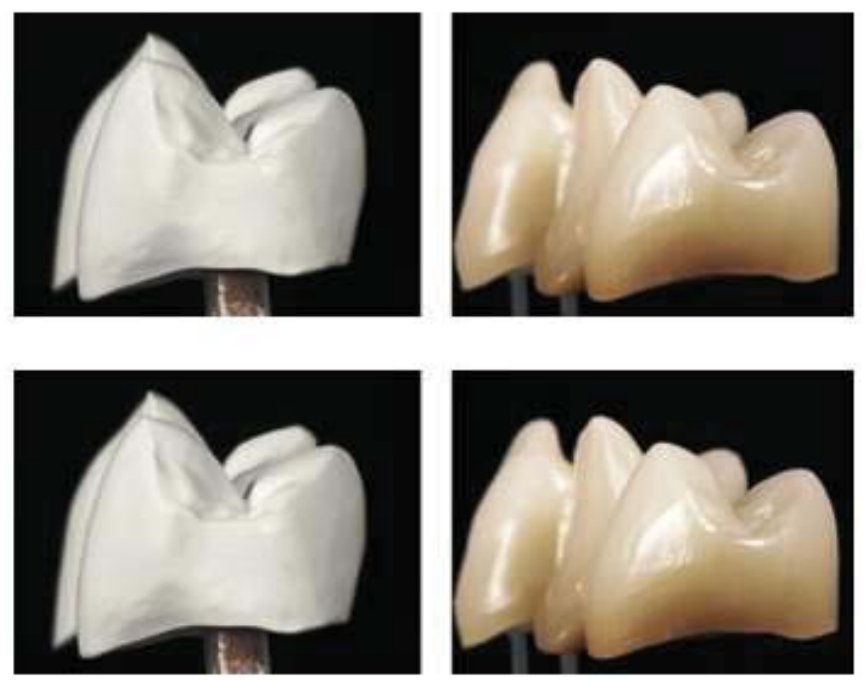

Figura 7. Coronas monolíticas de Y-TZP antes de sinterizado (izquierda) y después del sinterizado y maquillado (derecha) (7).

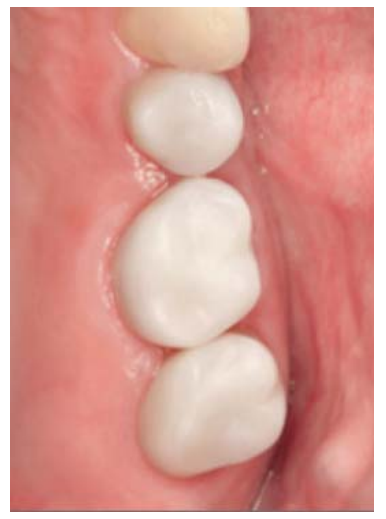

Figura 8. Coronas monolíticas de Y-TZP translúcidas (7).
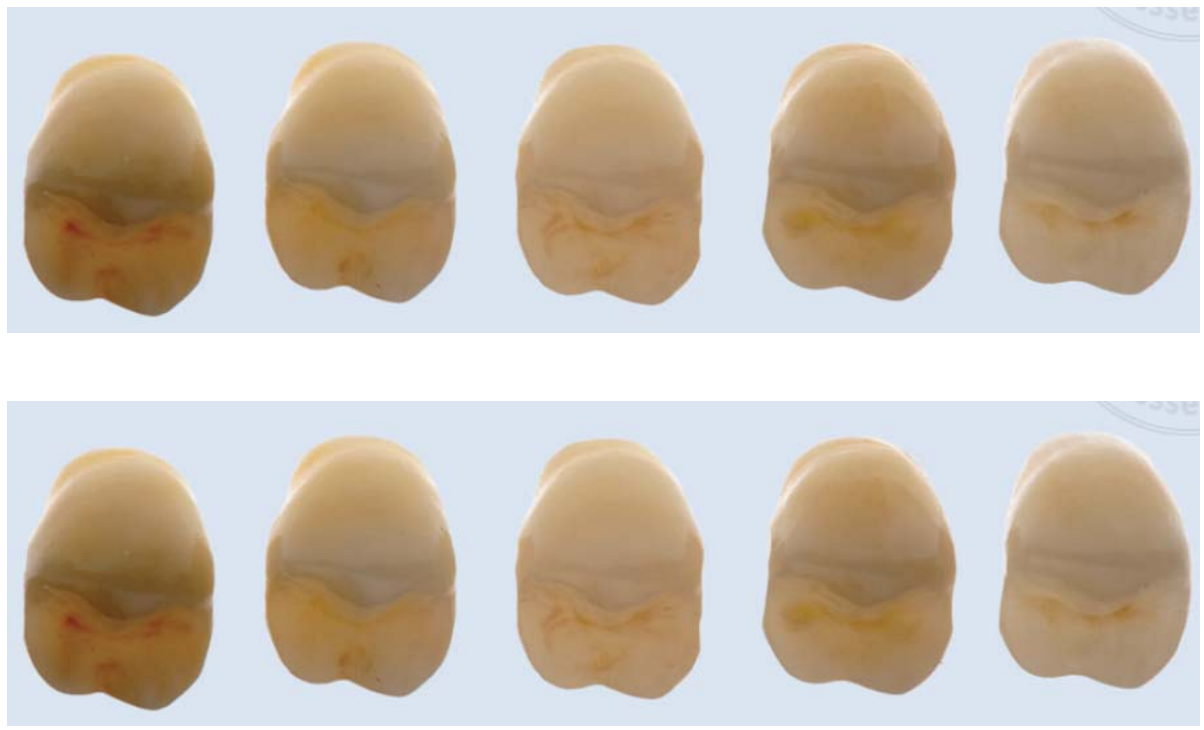

Figura 9. Comparación de las diferentes generaciones de zirconia comparado con el disilicato de litio (derecha a izquierda: $1^{\circ}$ generación, $2^{\circ}$ generación, $3^{\circ}$ generación, disilicato de litio LT y disilicato de litio HT) (8).

en relación con la segunda generación, conduce a una disminución en la resistencia del material $(2,5$, $8)$, existe literatura controversial con este punto, ya que investigadores mencionan que esta disminución no debería afectar su utilización, ya que resultados muestran que con zirconia de tercera generación los valores de resistencia a la fractura aún son superiores a la los valores máximos encontrados en la región posterior $(\approx 900 \mathrm{~N})(11)$, es así, que debido a la evidente disminución de la resistencia mecánica del material, la cual era la mayor ventaja del dióxido de zirconio, se decide seguir realizando alteraciones en su composición química hasta mejorar el balance entre la estética y mecánica, naciendo de esta manera, la zirconia de cuarta generación (4Y-TZP), donde, en relación a la generación anterior, trataron de 
disminuir el $\mathrm{A} 12 \mathrm{O} 3$ de $10 \%$ a $6 \%$ y aumentar el $\mathrm{Zr} 2 \mathrm{O}$ de $90 \%$ a $92 \%$, traduciéndose en el aumento de la fase tetragonal y disminución de la cúbica $(75 \%$ y $25 \%$ respectivamente) $(6,12)$. Este material presenta una mejora en sus propiedades mecánicas, pero sigue manteniendo las indicaciones de coronas unitarias y monolíticas preferentemente (11).

Es así, que la introducción del Dióxido de Zirconio Monolítico Translúcido resulta en dos importantes rangos de aplicación, como son: Optimización estética de restauraciones estratificadas con estructura anatómica (referidas al maquillaje superficial si fuese necesario) y resistencia en coronas y prótesis parciales fijas en la región posterior o de tramos largos (enfocadas a la no disminución clínica significativa de la resistencia del zirconio) $(5,7,8)$.

\section{Diseño de la Estructura Anatómica para la modificación del Zirconio Translúcido}

Los resultados de los estudios clínicos demuestran que un pronunciado diseño de la estructura anatómica y un prolongado enfriamiento conducen a una significativa reducción de las tasas de fractura, además de mejorar las propiedades ópticas; y en el caso de revestirla con cerámica, las capas aplicadas manualmente de las restauraciones de zirconio en la región molar se comportan mejor ante fuerzas compresivas $(2,8)$.

\section{Optimización estética de las Restauraciones Anteriores}

El incremento de la transmisión de la luz del zirconio modificado con translucidez mejora los resultados estéticos en estas áreas, siempre y cuando las preparaciones no presenten decoloraciones que requieran enmascaramiento o tengan postes metálicos. Estas modificaciones no influyen en las propiedades mecánicas de la restauración. En casos con limitado espacio disponible, es posible fabricar la restauración solo con recubrimiento vestibular $(5,8)$.

\section{DISCUSIÓN}

En lo que respecta al ajuste marginal Brawek et al., concluyeron que el ajuste marginal en restauraciones cerámicas fabricadas en sistemas CAD / CAM deberían ser menores a $120 \mu \mathrm{m}$ que era el valor aceptado desde 1971 con McLean, dando un rango permisible de 49 a $100 \mu \mathrm{m}$ (12). Estudios recientes con el avance de los centros de frezado nos muestran que el ajuste marginal depende, dentro de otros factores, del tipo de impresión, donde observamos que la impresión tradicional (polivinilsiloxano) aún conserva cierta ventaja sobre las computarizadas (scaners intraorales)(13); pero, otro investigadores mencionan que es posible que los resultados hayan diferido debido a la experiencia de los operadores, técnicos de laboratorio e incluso el método de medición de dichas discrepancias, aduciendo que ambas técnicas deberían tener resultados similares con una buena aplicación y entrenamiento $(6,9)$. Los resultados que se obtuvieron al evaluar prótesis parciales fijas de hasta tres unidades, mostraron que la adaptación marginal varía dependiendo de la técnica utilizada pero no es significativa entre técnicas e intra técnicas $(4,14)$. Existe mucha discrepancia en los resultados obtenidos por los diferentes autores, incluso al evaluar las justificaciones de los resultados, eligen interpretarlos de diferentes maneras, enfatizando variables que no se repiten entre investigadores. Por otro lado, Min-Kyung et al., compararon el ajuste marginal del disilicato de litio, zirconia monolítica opaca y translúcida; encontrando un mejor ajuste para el disilicato de litio seguido de la zirconia translúcida, además de concluir que el ajuste marginal no estaba en relación al tipo de terminación (15); pero en otro estudio con una metología similar, encontraron que si bien no existía diferencia significativa en el desajuste marginal y oclusal, sí se observaban menores desajustes axiales cuando se usaban técnicas digitales de impresión $(8,16,17)$, en este punto se puede evidenciar que si bien los ajustes marginales están, en su mayoría dentro de los aceptables clínicamente, aún se continúa con la búsqueda de disminuir más estos valores con la finalidad, quizá de obtener una pasividad real entra la restauración y el sustrato, aunque otras variables también entran a tallar en este punto.

Amir et al., observaron que las coronas de zirconia monolíticas tienen mayor resistencia a la fractura en comparación a las coronas con recubrimiento feldespático y disilicato de litio monolítico (18), pero en otra investigación tuvieron valores poco menores que las metal-cerámicas $(2,11)$. Al comparar las coronas monolíticas de zirconia opaca y translúcida se evidenció que el grupo translúcido obtenía mejores resultados de resistencia a la fractura, pero al comparar las mismas con porcelana de recubrimiento se observó lo contrario, aunque incluso así obtenían mejores resultados que las coronas monolíticas 
de disilicato de litio $(5,8,13)$, pero autores como Elsayed y col.10 muestran evidencia que el aumento de óxido de itrio a las coronas para favorecer su translucidez, genera una disminución en la resistencia flexural, traccional y a la fractura comparable al disilicato de litio, pero superando por mucho las fuerzas masticatorias máximas ejercidas. Otro estudio, enfocado en el sector anterior para evaluar la resistencia en función a vectores de fuerza diferentes concluyó que las coronas monolíticas de disilicato de litio tenían mejores propiedades, en lo que refiere a resistencia debido al módulo de elasticidad, que las de zirconia con recubrimiento y comparables a las monolíticas $(3,19)$. Para la evaluación de la resistencia a la fractura in vitro debemos saber que no son totalmente determinantes por la variedad y dirección de fuerzas que encontramos en la cavidad oral, entonces muchos investigadores prefieren solo tenerlo como dato estadístico (16); pero la justificación para estos estudios se basa en el método de termociclado para simular el envejecimiento a las restauraciones y, poder así tener resultados que puedan extrapolarse a las situaciones clínicas $(3,12$, 14), entonces para cuestiones prácticas Weigl et al., observaron que estructuras de zirconia de $0.5 \mathrm{~mm}$ son capaces de soportar elevadas tensiones con y/o sin termociclado (2), incluso algunas estructuras de $0,2 \mathrm{~mm}$ fueron capaces de soportar fuerzas por encima de las masticatorias, aunque en otros estudios se concluía que son necesarios al menos $0,6 \mathrm{~mm}$ para que puedan resistir las fuerzas masticatorias; este punto a evaluar sobre la resistencia a la fractura $y / 0$ flexión de material es concluyente para afirmar que las restauraciones a base de zirconia son las que mejor pronóstico van a presentar a lo largo del tiempo en cuestiones mecánicas por su composición estructural, y aunque se le han ido agregando otros componentes para optimizar la parte estética, aún se puede observar que son recomendables por su resistencia que es mayor a las fuerzas masticatorias promedio.

Si evaluamos a las coronas monolíticas en relación al desgaste (término para describir la pérdida progresiva de la superficie de un diente debido a varios factores, excepto la caries dental)(16), que pueden ocasionar a las estructuras antagonistas, encontramos estudios con diversos resultados y conclusiones divergentes por la suma de variables consideradas, básicamente algunos mencionan que va a depender de la metodología del estudio (in vitro/in vivo) $(12,18)$, otros el tipo de antagonista a utilizarse (esmalte, hidroxiapatita reforzada, acero inoxidable, resina acrílica, cerámica, etc.) $(9,17)$, pero al parecer convergen en el punto de que la restauración va a ocasionar mayor desgaste que un antagonista natural $(13,16)$.

El desgaste superficial de las cerámicas al darse por un mecanismo de microfractura, difieren de las resinas que se desgastan a través de abrasión por fatiga (14), según algunos investigadores la resistencia a la fractura y la rugosidad de superficie son las razones principales para el desgaste (12), más no los valores de dureza $(7,13)$; Alfrisany et al., encuentran que las superficies de zirconia causan menos desgaste a las superficies de esmalte antagonista en comparación al ocasionado por cerámica feldespática, pero recomienda que las superficies de zirconia no deben ser glaseadas, ya que, cuando comparan las superficies solo con acabado y pulido ocasionan menos desgaste que cuando están glaseadas (20), aunque otros estudios muestran que no hay diferencia estadísticamente significativa entre ambos $(8,12)$; cabe mencionar que si bien el glaseado es un método para brindar a la superficie de la restauración un alto brillo, las capas pueden desgastarse dentro de los 6 meses posteriores a la restauración. Para otros autores, el procedimiento de pulido, no agrega ninguna capa a la superficie de dicha restauración, entonces en términos de corto plazo la superficie glaseada lucirá como una superficie sin acabado ni pulido, ocasionando mayores desgastes (11). Pereira et al., argumentan en favor de la zirconia, en su estudio observan que el motivo de que las zirconias monolíticas, causan menos desgaste al antagonista es por su resistencia al micro desprendimiento de partículas durante la fricción, lo que no ocurre con cerámicas menos resistentes, que ante la constante fricción sufre astillamientos superficiales microscópicos, que son los causantes del desgaste del esmalte dental, entonces el desgaste a la fricción no tiene nada que ver con la resistencia mecánica del material como se creía anteriormente (21). Mai et al., compararon cuatro tratamientos superficiales combinando los protocolos de acabo y pulido, confirmando la necesidad de una secuencia protocolar entre aditamentos para reducir la rugosidad superficial del material y evitar consecuencias como adherencia bacteriana y desgastes mayores a antagonistas (9); resultados similares obtuvieron Caglar et al., (1); en todo caso Lee et alrelacionaron el procedimiento de acabado y pulido con una pequeña 
cantidad de fase monoclínica en la zirconia por la utilización de diferentes fresas de diamante(aumento de temperatura), pero al parecer no produciría cambios significativos en la resistencia del material (22). Habib et al., en contra parte a los otros autores antes mencionados, evaluaron la rugosidad superficial después de cargas cíclicas, responsable directa del desgaste, observando que la restauración metalcerámica y zirconia son las que menos rugosidad superficial presentaban después de las cargas cíclicas, afirmando que si bien las muestras metalcerámicas y zirconias no genera demasiada rugosidad superficial, pueden ocasionar mayor desgaste vertical del antagonista (11). Desde el inicio de la utilización del dióxido de zirconio se ha planteado que al ser el más resistente, ocasionaba mayor desgaste a piezas antagonistas, pero poco a poco se ha ido aclarando ese tema en favor de las restauraciones con dióxido de zirconio, aunque en este punto la metología es una variable sensible debido a las múltiples formas en que se trabaja la zirconia, adicionando el protocolo clínico a seguir.

Un punto muy discutido e importante acerca del dióxido de zirconio, es la cementación; es así que a los largo de los años han surgido muchas recomendaciones para la adhesión, unas más utilizadas y confiables que otras; empezando de la premisa que es una cerámica ácido resistente que no se puede grabar como las que contienen matriz vítrea (silicatos, polímeros e híbridos) (23). Los protocolos más aceptados utilizan el arenado con dióxido de aluminio con diferentes tamaños de granos, y se ha observado que mientras mayor el tamaño del grano, podría afectar la estructura tridimensional de la zirconia y sufrir daños que conlleven a la futura fractura (24); posteriormente, la búsqueda de unión química y/o micromecánica entre la estructura de zirconia y el sustrato dental, ha sido tema de discusión, ya que existen investigares que insisten en tratarlas como una cerámica vítrea, utilizando el "arenado triboquímico" buscando silicatizar las superficies de zirconia para utilizar procedimientos adhesivos convencionales $(25,26)$; aunque no se ha demostrado una mayor adhesión utilizando este protocolo en comparación a otros sin la utilización del arenado triboquímico (27). Se ha demostrado que el protocolo con mayor aceptación para la cementación de restauraciones de dióxido de zirconio es utilizando, posterior a un arenado, un primer a base de 10-MDP para la restauración; y para el sustrato dental, un cemento resinoso autoadhesivo $(28,29)$. En la literatura, también se compara otro tipo de agentes de unión, diferentes a los resinosos para la cementación de restauraciones a base de zirconia, desde el fosfato de zinc hasta los ionómeros, donde observamos que un factor fundamental para el éxito de cementos no resinosos, es la preparación geométrica del muñón, mientras más retentiva sea, se pueden obviar los procedimientos de retención et al., evaluaron la tasa de éxito de coronas cementadas con fosfato de zinc y un cemento de resina autoadhesivo y observaron una tasa de fracaso del $12,5 \%$ y $6,6 \%$ respectivamente, concluyendo que el cemento resinoso es más adecuado para estos casos (29). Sax et al., al evaluar la supervivencia de prótesis fijas de 3 unidades en un tiempo de 10 años cementados con protocolos autoadhesivos, encuentran una tasa de supervivencia del $67 \%$ para restauración y del $91.5 \%$ para la cofia de zirconia (30); lo contrario sucede en el estudio de Sorrentino et al., donde observan que la supervivencia en 5 años fue del $100 \%$ y el éxito de $95.4 \%$, ambos estudios reflejan ciertas antítesis de resultados, aunque tienen metodologías similares, la explicación de los fallos podría atribuirse a la manufactura mediante $\mathrm{CAD} / \mathrm{CAM}$, como a la convencional durante el recubrimiento, más que al tipo de cemento utilizado, ya que en ambos se usaron cementos autoadhesivos de resina (31).

Finalmente, podemos afirmar que las coronas monolíticas de Y-TZP (con todas sus varientes) pueden ser una alternativa de tratamiento prometedor, dependiendo de la situación clínica después de un exhaustivo análisis de los requerimientos mecánicos y/o estéticos del paciente, especialmente para aquellos con un historial de restauraciones fracturadas, bruxistas, expectativas estéticas altas, disminución de dimensión vertical, desgaste extrínseco o intrínseco y oclusión desfavorable. Por otra parte, las propiedades del material de alta translucidez (4Y-TPZ y 5Y-TZP) deben continuar siendo investigadas y corroboradas con ensayos clínicos a largo plazo, por lo pronto los resultados son alentadores y muy similares al dióxido de zirconio convencional (3Y-TZP) en cuestiones mecánicas y sin cambios significativos en términos clínicos.

\section{CONCLUSIONES}

El dióxido de zirconio se presenta como el material 
cerámico con mejores propiedades mecánicas $\mathrm{y}$, con las recientes modificaciones estructurales, se perfila a mejorar sus propiedades estéticas, presentando una solución al problema de opacidad que se observaba en las restauraciones monolíticas; este material que está en constante crecimiento se debe mucho también al agregado de aditivos, especialmente el óxido de itrio, que logró estabilizarlo molecularmente, sin disminuir sus propiedades mecánicas significativamente, especialmente como restauración monolítica.

Es más ventajoso el uso de zirconia en comparación a otro tipo de cerámica en cuestiones de desgaste del antagonista, pero el ajuste marginal aún puede ser un inconveniente en las restauraciones fabricadas mediante tecnología $\mathrm{CAD} / \mathrm{CAM}$ en comparación con las cerámicas inyectadas.

El dióxido de zirconio al no ser una cerámica vítrea, no puede grabarse para generar retenciones internas, el protocolo más difundido es el llamado APC (arenado, primer y cemento autoadhesivo) el cual ha demostrado resultados prometedores a mediano y largo plazo, además también se debe tener en cuenta la geometría del pilar y la altura para favorecer al adhesión.

Actualmente contamos con muchos tipos y generaciones de dióxido de zirconio, que dependiendo a la necesidad y finalidad de tratamiento deberán ser elegidas, ya que no todos poseen las mismas propiedades y características, e incluso una misma generación puede variar de acuerdo al fabricante.

Conflicto de intereses: Los autores declaran que no tienen conflicto de intereses.

\section{Correspondencia:}

Jesús Marcelo Ingunza

Correo electrónico: jesus.marcelo@upch.pe

\section{REFERENCIAS BIBLIOGRÁFICAS}

1. Caglar I, Ates SM, Yesil Z. The effect of various polishing systems on surface roughness and phase transformation of monolithic zirconia. J Adv Prosthodont. 2018; 10(2):132-137. doi: 10.4047/ jap.2018.10.2.132

2. Weigl P, Sander A, Wu Y, Felber R, Lauer HC, Rosentritt M. In-vitro performance and fracture strength of thin monolithic zirconia crowns. J Adv Prosthodont. 2018; (2):79-84. doi: 10.4047/jap.2018.10.2.79

3. Gunge H, Ogino Y, Kihara M, Tsukiyama Y, Koyano K. Retrospective clinical evaluation of posterior monolithic zirconia restorations after 1 to 3.5 years of clinical service. J Oral Sci. 2018; 60(1):154-158. doi: 10.2334/josnusd.17-0176.

4. Johansson C, Kmet G, Rivera J, Larsson C, VonSteyern P. Fracture strength of monolithic all-ceramic crowns made of high translucent yttrium oxidestabilized zirconium dioxide compared to porcelainveneered crowns and lithium disilicate crowns. Acta Odontol. Scand. 2014; 72(2): 145-53. doi: 10.3109/00016357.2013.822098

5. Lawson N, Burgess J. Dental Ceramics: A Current Review. Compend Contin Educ Dent. 2014; 35(3):161-6.

6. Miyazaki T, Nakamura T, Matsumura H, Ban S, Kobayashi T. Current status of zirconia restoration. J Prosthodont Res. 2013; 57(4): 236-61. doi: 10.1016/j. jpor.2013.09.001

7. Rinke S, Fischer C. Range of indications for translucent zirconia modifications: clinical and technical aspects. Quintessence Int. 2013; 44(8):557-66. doi: 10.3290/j. qi.a29937

8. Stawarczyk B, Keul C, Eichberger M, Figge D, Edelhoff D, Lümkemann N. Three generations of zirconia: From veneered to monolithic. Part I. Quintessence Int. 2017; 48(5):369-380. doi: 10.3290/j. qi.a38057

9. Mai HN, Hong SH, Kim SH, Lee DH. Effects of different finishing/polishing protocols and systems for monolithic zirconia on surfacetopography, phase transformation, and biofilm formation. J Adv Prosthodont. 2019; 11(2):81-7. doi: 10.4047/ jap.2019.11.2.81.

10. Elsayed A, Meyer G, Wille S, Kern M. Influence of the yttrium content on the fracture strength of monolithic zirconia crowns after artificial aging. Quintessence Int. 2019; 50(5):344-348. doi: 10.3290/j.qi.a42097

11. Habib SR, Alotaibi A, Al-Hazza N, Allam Y, Al-Ghazi M. Two-body wear behavior of human enamel versus monolithic zirconia, lithium disilicate, ceramometal and composite resin. J Adv Prosthodont. 2019; 11(1):23-31. doi: 10.4047/jap.2019.11.1.23

12. Brawek PK, Wolfart S, Endres L, Kirsten A, Reich $\mathrm{S}$. The clinical accuracy of single crowns exclusively fabricated by digital workflow-the comparison of two systems. Clin Oral Invest 2013; 17(9):2119-25. doi: 10.1007/s00784-013-0923-5

13. Moustapha G, Azzam K, AlShwaimi E, Silwadi M, Ferrari M, Salameh Z. Evaluation of the fit of zirconia three-unit fixed partial dentures fabricated by different impression techniques. J Investig Clin Dent. 2019; 10(3):e12413. doi: 10.1111/jicd.12413 
14. Schönberger J, Erdelt KJ, Bäumer D, Beuer F. Marginal and internal fit of posterior three-unit fixed zirconia dental prostheses fabricated with two different CAD/ CAM systems and materials. Clin Oral Investig. 2017; 21(8):2629-2635. doi: 10.1007/s00784-017-2064-8

15. Min-Kyung J, Ji-Hee P, Sang-Won P, Kwi-Dug Y, Gye-Jeong O, Hyun-Pil L. Evaluation of marginal fit of 2 CAD-CAM anatomic contour zirconia crown systems and lithium disilicate glass-ceramic crown. J Adv Prosthodont. 2015; 7(4):271-7. doi: 10.4047/ jap.2015.7.4.271

16. Al Hamad KQ, Al Quran FA, AlJalam SA, Baba NZ. Comparison of the Accuracy of Fit of Metal, Zirconia, and Lithium Disilicate Crowns Made from Different Manufacturing Techniques. J Prosthodont. 2019; 28(5):497-503. doi: 10.1111/jopr.13029

17. Benic GI, Sailer I, Zeltner M, Gütermann JN, Özcan M, Mühlemann S. Randomized controlled clinical trial of digital and conventional workflows for the fabrication of zirconia-ceramic fixed partial dentures. Part III: Marginal and internal fit. J Prosthet Dent. 2019; 121(3):426-431. doi: 10.1016/j.prosdent.2018.05.014.

18. Amir Rad F, Succari F, Morgano S. Fracture resistance of porcelain veneered zirconia crowns with exposed lingual zirconia for anterior teeth after thermal cycling: An in vitro study. Saudi Dent J. 2015; 27(2):63-9. doi: 10.1016/j.sdentj.2014.11.005.

19. Miura S, Kasahara S, Yamauchi S, Egusa H. Effect of finish line design on stress distribution in bilayer and monolithic zirconia crowns: a three-dimensional finite element analysis study. Eur J Oral Sci. 2018; 126(2):159-165. doi: 10.1111/eos. 12402.

20. Alfrisany NM, Shokati B, Tam LE, De Souza GM. Simulated occlusal adjustments and their effects on zirconia and antagonist artificial enamel. J Adv Prosthodont. 2019; 11(3):162-168. doi: 10.4047/ jap.2019.11.3.162

21. Pereira GKR, Dutra DM, Werner A, Prochnow C, Valandro LF, Kleverlaan CJ. Effect of zirconia polycrystal and stainless steel on the wear of resin composites, dentin and enamel. J Mech Behav Biomed Mater. 2019; 91:287-293. doi: 10.1016/j. jmbbm.2018.12.024.

22. Lee KR, Choe HC, Heo YR, Lee JJ, Son MK. Effect of different grinding burs on the physical properties of zirconia. J Adv Prosthodont. 2016; 8(2):137-43. doi: 10.4047/jap.2016.8.2.137

23. Chen YW, Moussi J, Drury JL, Wataha JC. Zirconia in biomedical applications. Expert Rev Med Devices. 2016; 13(10):945-63. doi: 10.1080/17434440.2016.1230017

24. Schünemann FH, Galárraga-Vinueza ME, Magini $\mathrm{R}$, et al. Zirconia surface modifications for implant dentistry. Mater Sci Eng C Mater Biol Appl. 2019;
98:1294 -305. doi: 10.1016/j.msec.2019.01.062

25. Papia E, Larsson C, du-Toit M, Vult-von-Steyern P. Bonding between oxide ceramics and adhesive cement systems: a systematic review. J Biomed Mater Res B Appl Biomater. 2014; 102(2):395-413. doi: 10.1002/ jbm.b.33013

26. Özcan M, Bernasconi M. Adhesion to zirconia used for dental restorations: a systematic review and meta-analysis. J Adhes Dent. 2015; 17(1):7-26. doi: 10.3290/j.jad.a33525

27. Dal-Piva AMO, Carvalho RLA, Lima AL, Bottino MA, Melo RM, Valandro LF. Silica coating followed by heat-treatment of MDP-primer for resin bond stability to yttria-stabilized zirconia polycrystals. J Biomed Mater Res B Appl Biomater. 2019; 107(1):104-11. doi: 10.1002/jbm.b.34100

28. Blatz MB, Alvarez M, Sawyer K, Brindis M. How to bond to zirconia: the APC concept. Compend Contin Educ Dent. 2016; 37(9):611-618..

29. Ortorp A, Kihl ML, Carlsson GE. 2012. A 5-year retrospective study of survival of zirconia single crowns fitted in a private clinical setting. J Dent. 40(6):527-530. doi: 10.1016/j.jdent.2012.02.011

30. Sax C, Hämmerle CH, Sailer I. 10-year clinical outcomes of fixed dental prostheses with zirconia frameworks. Int J Comput Dent. 2011; 14(3):183202. doi: 10.5167/uzh-57076

31. Sorrentino R, De-Simone G, Tetè S, Russo S, Zarone F. Five-year prospective clinical study of posterior three-unit zirconia-based fixed dental prostheses. Clin Oral Investig. 2012; 16(3):977-985. doi: 10.1007/ s00784-011-0575-2

Recibido: 13-02-19

Aceptado: 25-06-20 